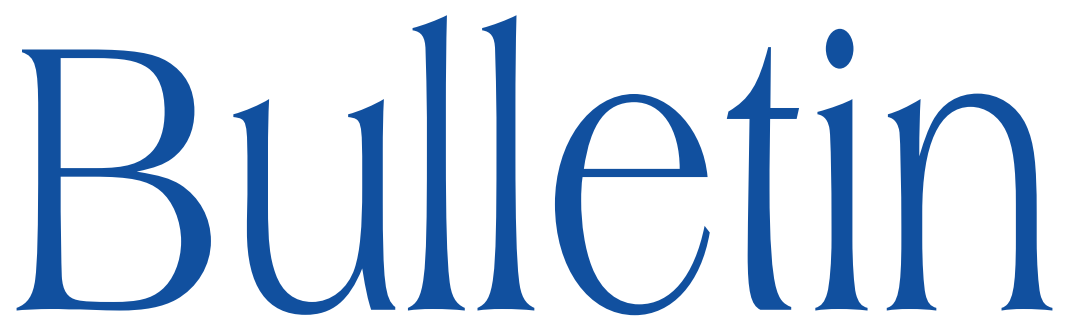

de la SOCIÉTÉ MATHÉMATIQUE DE FRANCE

\title{
ON THE RIGIDITY OF WEBS
}

\author{
Michel Belliart
}

\section{Tome 135} Fascicule 1

2007

\section{SOCIÉTÉ MATHÉMATIQUE DE FRANCE}


Bull. Soc. math. France

135 (1), 2007, p. 1-24

\title{
ON THE RIGIDITY OF WEBS
}

\author{
BY MiCHEL BELLIART
}

\begin{abstract}
Plane $d$-webs have been studied a lot since their appearance at the turn of the $20^{\text {th }}$ century. A rather recent and striking result for them is the theorem of Dufour, stating that the measurable conjugacies between 3-webs have to be analytic. Here, we show that even the set-theoretic conjugacies between two $d$-webs, $d \geq 3$ are analytic unless both webs are analytically parallelizable. Between two set-theoretically conjugate parallelizable $d$-webs, however, there always exists a nonmeasurable conjugacy; still, every pair of set-theoretically conjugate 3-webs (parallelizable or not) also are analytically conjugate, while if $d \geq 4$ there exist pairs of $d$-webs which are settheoretically conjugate but not even measurably so.

RÉsumé (Sur la rigidité des tissus). - Les $d$-tissus plans ont été amplement étudiés depuis leur apparition au début $\mathrm{du} \mathrm{xx}^{\mathrm{e}}$ siècle. Un résultat relativement récent et impressionnant est le théorème de Dufour qui stipule que les conjugaisons mesurables entre 3-tissus sont nécessairement analytiques. Dans cet article nous montrons que les conjugaisons ensemblistes entre $d$-tissus (avec $d \geq 3$ ) sont analytiques sauf si les deux tissus sont analytiquement parallélisables. Cependant, entre deux $d$-tissus parallélisables conjugués de manière ensembliste il existe toujours une conjugaison nonmesurable; de plus, toute paire de 3-tissus conjugués de manière ensembliste (qu'ils soient parallélisables ou non) sont également conjugués analytiquement, alors que si $d \geq 4$, il existe des paires de $d$-tissus qui sont conjugués de manière ensembliste mais non pas de manière mesurable.
\end{abstract}

Texte reçu le 4 octobre 2004, révisé le 28 juin 2006

Michel Belliart, UMR AGAT, UFR de Mathématiques, Université Lille I, 59655 Villeneuve d'Ascq Cedex (France) • E-mail : belliart@agat.univ-lille1.fr

2000 Mathematics Subject Classification. - 53A60, $22 \mathrm{~F} 05$.

Key words and phrases. - Foliations, 3-webs, conjugacy, rigidity. 


\section{Introduction}

1.1. Foliations. - Throughout this note we consider the word "foliation" as meaning "analytic foliation of the plane $\mathbb{R}^{2}$ by curves". The classification of foliations (in this very restricted sense of the word) has been known for years; first of all, we can describe foliations in two dual ways:

- Given any foliation $\mathcal{F}$, there exists an analytic action of $\mathbb{R}$ on $\mathbb{R}^{2}$ whose orbits are the leaves of $\mathcal{F}$ (this is not difficult at all to show).

- Given any foliation $\mathcal{F}$, there exists a continuous submersion $\phi$ from $\mathbb{R}^{2}$ to $\mathbb{R}$ which is constant along the leaves of $\mathcal{F}$ : therefore these leaves are the connected components of the fibers of $\phi$ (this is Kaplan's Theorem [9]).

Starting from there, it is not too difficult to describe the topological conjugacy classes of foliations; see [7] for a nice description of the classification (due to Kaplan [10]).

We simply define the set-theoretic conjugacies between two foliations $\mathcal{F}$ and $\mathcal{F}^{\prime}$ as being bijective maps from $\mathbb{R}^{2}$ to $\mathbb{R}^{2}$ which send every leaf of $\mathcal{F}$ bijectively onto some leaf of $\mathcal{F}^{\prime}$. This is a straightforward but completely formal definition; observe that any two foliations are set-theoretically conjugate! But the following very simple example shows us that the notion of a set-theoretic conjugacy already stops being trivial if we consider more than just one foliation at a time.

EXAMPLE 1.1.1. - Let $\mathcal{F}_{1}$ be the foliation of $\mathbb{R}^{2}$ by horizontal lines; let $\mathcal{F}_{2}$ be that by vertical lines, and let $\mathcal{F}_{3}$ be that by curves having the form $y=\mathrm{e}^{x}+C$ where $(x, y)$ are the natural coordinates and $C$ is a parameter. Then, there is no bijection of $\mathbb{R}^{2}$ onto $\mathbb{R}^{2}$ inducing a conjugacy of $\mathcal{F}_{1}$ onto itself and a conjugacy of $\mathcal{F}_{2}$ onto $\mathcal{F}_{3}$ at the same time: indeed any leaf of $\mathcal{F}_{1}$ and any leaf of $\mathcal{F}_{2}$ meet at exactly one point, while for every leaf $L$ of $\mathcal{F}_{3}$ there is on the contrary a leaf of $\mathcal{F}_{1}$ not meeting $L$.

1.2. Webs. - Funnily enough, webs have appeared in mathematics way before foliations, perhaps because their local geometry is so visibly richer. We call $d$ web the datum $\mathcal{W}=\left(\mathcal{F}_{1}, \ldots, \mathcal{F}_{d}\right)$ of $d \geq 3$ foliations which we require to be pairwise transverse at each point: this means e.g., that any leaf $L_{1}$ of $\mathcal{F}_{1}$ and any leaf $L_{2}$ of $\mathcal{F}_{2}$, if not disjoint, intersect at exactly one point with distinct tangents.

We should mention that usually, a web is locally defined as an unordered collection of $d$ foliations (possibly singular) which are in general position. There are then obstructions to the possibility of "separating" this data in $d$ distinct foliations; but these obstructions read either on the singular locus of the configuration, or on the topology of the ambient manifold. Here, we chose to work with everywhere transverse nonsingular (local) foliations of a contractible 
space; for this reason, our $d$ local foliations glue into global ones and we can order the collection of them.

In 1908 already, Cartan [3] asks to study the topology of the figure formed by three families of curves, a problem which he calls the first problem of textile geometry. From the 1930ies on, the school of Blashke and Bol will give this problem due consideration, as well as generalize it a lot; the works of that school are collected in the books [2] and [1]. For a more modern point of view on web theory, we should mention the surveys [4] and [13] written by two experts in the field.

We say, of course, that the bijection $f$ of $\mathbb{R}^{2}$ conjugates the $d$-web $\mathcal{W}$ to the $d$-web $\mathcal{W}^{\prime}$ if it conjugates each of the foliations forming $\mathcal{W}$ to the foliation of $\mathcal{W}^{\prime}$ which has the same index. Example 1.1.1 implies that not every couple of $d$-webs are conjugate in this way. Finally, we call a web parallelizable if it is conjugate to some web whose foliations are by parallel affine lines.

1.3. Rigidity. - We have grown used to the fact that a typical map from $\mathbb{R}$ to $\mathbb{R}$ is not measurable, that a typical measurable such map is not continuous, and so forth... But the first historical examples of such uncanny behaviour had been built as counterexamples and did not answer any "real" problems: for this reason, even after these first examples appeared in the works of Peano, Riemann, Weierstraß et al., one could still place some faith in the following informal, sadly erroneous belief,

Credo 1.3.1. - If a problem whose datum is purely analytic has a unique solution, then that solution must be analytic.

As we hinted to, the above credo was baffled by many counterexamples which, as a rule, came from dynamical systems theory: for instance, to certain dynamical systems - the so-called Anosov diffeomorphi sms - one can associate invariant foliations which will not necessarily be differentiable even if the diffeomorphism we started with is analytic (see [11, III.3]; we quote from the same source: "in general, even if $f$ is $C^{\infty}$, Poincaré transformations are not even Lipschitz"). Another famous example is that of the "cohomological equation" $f(x+k)-f(x)=g(x)$, where the datum $g$ and the unknown $f$ both are smooth functions from $\mathbb{R}$ to itself with period one and $k$ is a given real number: as soon as $k$ is irrational and $\int_{0}^{1} g(x) \mathrm{d} x=0$, there exists a formal solution for that equation in the shape of a Fourier series $\widehat{f}$ which, in general, does not converge at all; for $\widehat{f}$ to converge to a smooth function whatever our choice of $g$, the real number $k$ must be diophantine (see [8]). In conclusion, examples of analytic problems whose solutions only possess a low regularity abound in modern practice, and this is what makes the following result so very interesting to us: 
Theorem (Dufour, [6]). - Any measurable bijection of $\mathbb{R}^{2}$ onto itself which exchanges two 3-webs must be analytic.

This is the first version of Dufour's Theorem; we should perhaps mention the existence of a recent generalization of it to arbitrary dimensions and codimensions by the same author, and the existence of a complex version by Nakai in codimension one (see [5] and [12], respectively).

1.4. Statement of the theorems. - The purpose of this note is mostly to show:

Theorem 1.4.1. - Let $\mathcal{W}$ and $\mathcal{W}^{\prime}$ be two $d$-webs one of which at least is not analytically parallelizable. Then every set-theoretic conjugacy from $\mathcal{W}$ to $\mathcal{W}^{\prime}$ is analytic.

If $\mathcal{W}$ is a parallelizable web, one can easily show that $\mathcal{W}$ possesses nonmeasurable self-automorphisms; so, Theorem 1.4.1 is sharp. We should next wonder if the existence of a set-theoretic conjugacy $f$ between two $d$-webs always implies the existence of an analytic one $f^{\prime}$ not necessarily equal to $f$ : this is a weaker sort of rigidity. Theorem 1.4.1 already solved that problem except for parallelizable webs, for which we have

THEOREM 1.4.2. - The two notions of analytic conjugacy and of set-theoretic conjugacy for parallelizable $d$-webs are equivalent precisely if $d=3$.

For $d \geq 4$ we will exhibit interesting counterexamples.

We would like to underline the similarity of ideas between our proof of Theorem 1.4.1 and the so-called fundamental theorem of affine geometry. This famous result states that a bijection of $\mathbb{R}^{2}$ preserving the family of lines has to be affine; recall how the proof works: first, by using elementary constructions which are in fact valid over any field $\mathbb{K}$ but the field $\{0,1\}$, one associates to any line-preserving bijection $f$ of $\mathbb{K}^{2}$ a field automorphism $\tau_{f}$ of $\mathbb{K}$ such that the equality $f\left(\sum m_{\imath} M_{\imath}\right)=\sum \tau_{f}\left(m_{\imath}\right) M_{\imath}$ holds for any barycenter $\sum m_{\imath} M_{\imath}$ in $\mathbb{K}^{2}$. Thus, the map $f$ will be affine precisely if the field automorphism $\tau_{f}$ is trivial... which, as one knows, must happen if $\mathbb{K}=\mathbb{R}$. Now, the construction of $\tau_{f}$ uses the whole set of lines in $\mathbb{K}^{2}$; but if we restrict our attention to three particular families of parallel lines, we can still build an automorphism of abelian group $\tau_{f}$ of $\mathbb{K}^{2}$ and show that the equality $f(M+\vec{v})=f(M)+\tau_{f}(\vec{v})$ holds for every point $M$ and vector $\vec{v}$. This equality is a weakening of the former one obtained thanks to the whole set of lines, if we remember that formally, a vector is simply a barycenter with total mass zero. Instead of the fact that every field automorphism of $\mathbb{R}$ is trivial, we may now invoke the other fact that every measurable group automorphism of $\mathbb{R}^{2}$ is linear to obtain

TOME $135-2007-\mathrm{N}^{\mathrm{O}} 1$ 
what is both an alternative version of the fundamental theorem and a subcase of Dufour's Theorem:

THEOREM 1.4.3. - A measurable bijection from $\mathbb{R}^{2}$ to itself which preserves three directions of lines has to be affine.

As the reader will see, our proof of Theorem 1.4.1 consists in noticing that the proof to Theorem 1.4.3 which we just hinted to still works (and works even better!) if we replace our three directions of lines by a general $d$-web.

1.5. The method of proof. - Dufour shows his theorem using a certain normal form for 3-webs (the so-called Bol loop of the web). Nakai's proof is of a dynamical nature: although this is not exactly how this author puts it, the rigidity comes from the existence of a conjugacy between certain pseudogroups of biholomorphisms which are defined on the spaces of leaves of the involved complex webs. Our contribution to the subject is mostly to have seen that Nakai's construction, not only had nothing to do with holomorphy, but could even be performed by purely set-theoretical means and moreover yielded the continuity of the conjugating maps in most of cases.

In the second section of this paper, we deal with pseudoactions on curves in a somewhat abstract fashion; the main topic is the possibility of recovering the topology of the curve from the pseudoaction by purely set-theoretic means. In the third (and last) section, we study the geometry of $d$-webs; we start with the parallelizable case, which is quite elementary, and proceed by showing that general webs fall into three classes: A) incomplete webs, B) complete curved webs, and C) parallelizable webs. The words "complete" and "curved" are defined in Subsection 3.4; webs of the classes A and B are then shown to be rigid thanks to the main lemma of Section 2 which we apply to a certain pseudogroup that is built from the web in Subsection 3.3.

1.6. Generalizations and perspectives. - Because our object of study is so simple, we wished our statements to be simple too, and so we chose to work in the analytic category; we leave it to the reader to check that our methods work in fact for every class $C^{r}, r \in \mathbb{N} \cup\{\infty\}$ (and for other classes as well): that is, if we let the word "foliation" mean "foliation of $\mathbb{R}^{2}$ by level curves of a $C^{r}$ map", then Theorem 1.4.1 still holds, its original conclusion " $f$ is analytic" to be replaced with " $f$ is of class $C$ ".

One could next think of possible generalizations of Theorem 1.4.1 to webs with a "mixed" regularity (this was suggested by the referee). To mention one example that seems promising: consider webs whose global class is $C^{0}$, but whose leaves all are analytic. Our results only lead to the continuity of conjugacies (with the same exceptional case as in Theorem 1.4.1). But perhaps 
the conjugating map is analytic as soon as it is continuous? Our methods do not lead to this conclusion but we could think of no counterexample either.

It is the author's strong belief that webs are very rigid objects, and that this rigidity is worth exhibiting. The rigidity under consideration here is the strongest possible (no requirement is made at all on the map $f$ conjugating the given webs!) Then, the proofs deeply rely on the basic features of the topology of $\mathbb{R}^{2}$, so that it is highly improbable that Theorem 1.4.1 would extend to higher dimensions. But it seems to the author that a continuous (measurable?) bijection from a domain in $\mathbb{R}^{n}$ to another such domain which conjugates two families of analytic foliations may have to be analytic itself in certain privileged directions as soon as the two families of foliations under consideration are "general enough". Although no clear statement is available at the moment, there is the evidence of Dufour's and Nakai's higher dimensional results. This is a natural question to investigate. Also, because of the natural links between web theory and algebraic geometry, a wide part of web theory takes place in a local holomorphic context; it would therefore be interesting to investigate holomorphic webs from this point of view, in order to obtain generalizations of [13] (there again, it seems to the author that the involved conjugating maps should be required to be at least measurable; the dynamics of a complex onedimensional and codimensional foliation are much wilder than those of a real such foliation, and there is little hope that our set-theoretic approach would still work).

Acknowledgement. - The author wishes to thank heartfully Alain Hénaut, who read a first draft of this paper and made many very interesting comments on it, and also the referee of the first submitted version, for the same reasons.

\section{Curves and pseudogroups}

2.1. Curves. - In this section we will show a very simple lemma on the topology of a curve. Here, we call curve any topological space $\mathcal{C}$ whose connected components all are homeomorphic to $\mathbb{R}$; an interval in a curve $\mathcal{C}$ is for us any subspace homeomorphic to $\mathbb{R}$, and the interval is bounded (respectively bounded on one side) if it has two (respectively one) point(s) in its frontier. In particular, every interval is open in our terminology. There also is a natural notion of an orientation on a curve, and if $\mathcal{C}$ is endowed with one, the family of intervals which are bounded on one side naturally split into the two subfamilies of intervals unbounded to the left and intervals unbounded to the right. Here is the main example which we have in mind:

TOME $135-2007-\mathrm{N}^{\circ} 1$ 
ExAmPLE. - Let $\mathcal{F}$ be a foliation; we may find an analytic vector field $X$ everywhere tangent to $\mathcal{F}$ whose flow admits the leaves of $\mathcal{F}$ for its orbits (cf. Subsection 1.1). We now call interval of $\mathcal{F}$ any part of $\mathbb{R}^{2}$ which consists of all the points having the form $\exp (t X) m$ with $m$ a given point and $t$ varying in some open interval of $\mathbb{R}$; with this notion of an interval, $\mathbb{R}^{2}$ becomes a curve whose connected components are the leaves of $\mathcal{F}$. We write $\mathcal{C}(\mathcal{F})$ for that curve and call it the curve of $\mathcal{F}$. Our choice of $X$ provides us with a natural orientation of $\mathcal{C}(\mathcal{F})$, should we need one.

2.2. Convenient topologies on a curve. - From now on, let $\mathcal{C}$ be a curve. Let $\mathcal{T}_{1}(\mathcal{C})$ be its topology, and let $\mathcal{T}_{0}(\mathcal{C})$ be the (much weaker) topology on $\mathcal{C}$ whose open sets write as unions of connected components of $\mathcal{C}$ for $\mathcal{T}_{1}(\mathcal{C})$. Our interest lies in a certain type of topologies on $\mathcal{C}$, which are intermediary between $\mathcal{T}_{0}(\mathcal{C})$ and $\mathcal{T}_{1}(\mathcal{C})$ :

Definition 2.2.1. - A topology $\mathcal{T}$ on $\mathcal{C}$ is convenient if $\mathcal{T}_{0}(\mathcal{C}) \subset \mathcal{T}$ and if $\mathcal{T}$ is generated by intervals (so that $\mathcal{T} \subset \mathcal{T}_{1}(\mathcal{C})$ ).

EXAmPLe 2.2.2. - On $\mathcal{C}$, the topology whose nonempty open sets are the open neighborhoods of infinity is not convenient; indeed, unless $\mathcal{C}$ is connected, this topology contains no interval at all!

Definition 2.2.3. - Let $\vartheta$ denote some choice of an orientation on $\mathcal{C}$. We write $-\vartheta$ for the opposite orientation. We write $\mathcal{T}(\vartheta)$ for the convenient topology on $\mathcal{C}$ which is spanned by the intervals of $\mathcal{C}$ which are unbounded to the left (thus $\mathcal{T}(-\vartheta)$ stands for the convenient topology spanned by intervals which are unbounded to the right). Finally we let $\mathcal{G}(\mathcal{C})$ denote the space of "good" topologies on $\mathcal{C}$, which are by definition $\mathcal{T}_{1}(\mathcal{C})$ together with every topology having the form $\mathcal{T}(\vartheta)$.

Lemma 2.2.4. - Let $\mathcal{C}, \mathcal{C}^{\prime}$ be curves and let $f$ be a set-theoretic bijection from $\mathcal{C}$ to $\mathcal{C}^{\prime}$. If $f(\mathcal{G}(\mathcal{C})) \cap \mathcal{G}\left(\mathcal{C}^{\prime}\right) \neq \varnothing$ then $f(\mathcal{G}(\mathcal{C}))=\mathcal{G}\left(\mathcal{C}^{\prime}\right)$ and $f$ is a homeomorphism.

Proof. - It is obvious enough that the condition $f\left(\mathcal{T}_{1}(\mathcal{C})\right)=\mathcal{T}_{1}\left(\mathcal{C}^{\prime}\right)$ implies $f(\mathcal{G}(\mathcal{C}))=\mathcal{G}\left(\mathcal{C}^{\prime}\right)$. Next show that if $f(\mathcal{G}(\mathcal{C})) \cap \mathcal{G}\left(\mathcal{C}^{\prime}\right) \neq \varnothing$ then $f\left(\mathcal{T}_{1}(\mathcal{C})\right)=\mathcal{T}_{1}\left(\mathcal{C}^{\prime}\right)$. For that, consider some $\mathcal{T} \in \mathcal{G}(\mathcal{C})$; it suffices to show that we can build $\mathcal{T}_{1}(\mathcal{C})$ back from $\mathcal{T}$ by a purely set-theoretic construction. But we can describe $\mathcal{T}_{1}(\mathcal{C})$ as follows:

- If $\mathcal{T}$ is Hausdorff then $\mathcal{T}=\mathcal{T}_{1}(\mathcal{C})$.

- If not, then $\mathcal{T}_{1}(\mathcal{C})$ is the weakest topology having the following two properties, (a) $\mathcal{T} \subset \mathcal{T}_{1}(\mathcal{C})$ and (b) if $I_{n}$ is an infinite, strictly decreasing sequence 
of connected open sets for $\mathcal{T}$ then the complementary set of $\cap_{n} I_{n}$ in $\mathcal{C}$ is open for $\mathcal{T}_{1}(\mathcal{C})$.

2.3. Pseudogroups on a curve. - Let $\mathcal{C}$ still be a curve. Let $G$ be a collection of couples $(U, \phi)$ where $U$ is an interval in $\mathcal{C}$ and $\phi$ is an injective continuous map from $U$ to $\mathcal{C}$. We assume that $G$ has the following properties:

- Let $(U, \phi)$ belong to $G$ and let $V=\phi(U)$ : then the couple $\left(V, \phi^{-1}\right)$ lies in $G$.

- Let $(U, \phi)$ and $(V, \psi)$ belong to $G$ and let the interval $\phi(U) \cap V$ be nonempty in $\mathcal{C}$ : then, $\left(\phi^{-1}(\phi(U) \cap V), \psi \circ \phi\right)$ is in $G$.

Definition 2.3.1. - A collection of maps such as $G$ above we call a pseudogroup on $\mathcal{C}$ - keeping in mind that normally, a pseudogroup in the usual sense of the word should be required to satisfy two more axioms (stability by localization and by gluing) which we must forbear here, because they would ruin the proofs to come!

Here is a painstaking list of further definitions, holding no real surprise. First, given any $x$ in $\mathcal{C}$, we define the $G$-orbit of $x$ as the set of all $y$ in $\mathcal{C}$ for which there exists a $(U, \phi)$ in $G$ with $x \in U$ and $\phi(x)=y$; the relation of being in the same $G$-orbit is readily seen to be an equivalence relation on $\mathcal{C}$. Next, we call $G$ transitive on $\mathcal{C}$ if it has only one orbit. If $x$ is some point of $\mathcal{C}$, the stabilizer of $x$ in $G$ is the subset of couples $(U, \phi) \in G$ with $x \in U$ and $\phi(x)=x$; it is readily seen to be a subpseudogroup of $G$, i.e., a pseudogroup on $\mathcal{C}$ which is contained in $G$. There is an obvious notion of a $G$-invariant orientation on $\mathcal{C}$. Next, identifying an element $(U, \phi)$ of $G$ with its graph $\Gamma(U, \phi) \subset \mathcal{C} \times \mathcal{C}$, we can define the notion of a set-theoretic conjugacy $f$ between two pseudogroups $G$ and $G^{\prime}$ (the map $f$ is required to exchange the two collections of graphs corresponding to $G$ and $G^{\prime}$ ). Finally, call subcurve of $\mathcal{C}$ any subspace $\mathcal{C}^{\prime}$ of $\mathcal{C}$ which is a curve for the induced topology: then obviously, the collection $G\left(\mathcal{C}^{\prime}\right)$ of all couples $(U, \phi) \in G$ for which $U \subset \mathcal{C}^{\prime}$ and $\phi(U) \subset \mathcal{C}^{\prime}$ is a subpseudogroup of $G$ defined on $\mathcal{C}^{\prime}$. We call it the restriction of $G$ to $\mathcal{C}^{\prime}$.

2.4. Pseudogroups and convenient topologies. - Let still $\mathcal{C}$ be a curve and let $G$ be a pseudogroup on $\mathcal{C}$. Consider some topology $\mathcal{T}$ on $\mathcal{C}$.

Definition 2.4.1. - We say that $\mathcal{T}$ is invariant by $G$ if for any open set $O$ of $\mathcal{T}$ and any couple $(U, \phi)$ in $G$, the subset $\phi(U \cap O)$ of $\mathcal{C}$ is again an open set of $\mathcal{T}$. 
REMARK 2.4.2. - This condition implies in particular that for any $O$ and $(U, \phi)$ as above, $O \cap U$ belongs to $\mathcal{T}$ (indeed, a consequence of our two assumptions on $G$ is that the couple $\left(U, I_{U}\right)$ lies in $G$, where $I_{U}=\phi^{-1} \circ \phi$ denotes the identity map of $U$ ). As a special case, letting $O=\mathcal{C}$, we see that $U$ belongs to $\mathcal{T}$ for all $(U, \phi)$ in $G$.

Let $\mathcal{T}$ be a convenient topology on $\mathcal{C}$ which is invariant by $G$. Let $\Gamma$ be the set of all couples $(U, \phi)$ with the following properties: $U$ is an interval of $\mathcal{T}, \phi$ is an injective continuous map from $U$ to $\mathcal{C}$, and there exists a couple $\left(U^{\prime}, \phi^{\prime}\right)$ in $G$ with $U \subset U^{\prime}$ such that $\phi$ is the restriction of $\phi^{\prime}$ to $U$. Then, first of all, $\Gamma$ is a pseudogroup on $\mathcal{C}$; secondly, $\Gamma$ contains $G$; and lastly, the topology $\mathcal{T}$ is invariant by $\Gamma$.

Definition 2.4.3. - We call $\Gamma$ the completion of $G$ relative to $\mathcal{T}$.

EXAMPLE 2.4.4. - There are two very obvious convenient topologies on $\mathcal{C}$ which are invariant by every $G$ (they are $\mathcal{T}_{0}(\mathcal{C})$ and $\mathcal{T}_{1}(\mathcal{C})$ ). If we next endow $\mathcal{C}$ with an orientation $\vartheta$ and consider the pseudogroup $G$ consisting of all the couples $(U, \phi)$ such that $U$ is unbounded to the left, $\phi$ is a continuous orientation-preserving injection from $U$ to $\mathcal{C}$ and $\phi(U)$ is unbounded to the left, then $\mathcal{T}(\vartheta)$ will be invariant by $G$.

2.5. A lemma. - We now show:

Lemma 2.5.1. - Let $\mathcal{C}$ be a curve and let $G$ be a pseudogroup on $\mathcal{C}$; let $\mathcal{T}$ be a convenient topology on $\mathcal{C}$ which is invariant by $G$. We assume that $G$ preserves the orientation $\vartheta$ and is transitive on $\mathcal{C}$. Then

$$
\mathcal{T} \in\left\{\mathcal{T}_{0}(\mathcal{C}), \mathcal{T}_{1}(\mathcal{C}), \mathcal{T}(\vartheta), \mathcal{T}(-\vartheta)\right\}
$$

Proof. - Clearly, we can assume with no loss of generality that $G$ coincides with its completion relative to $\mathcal{T}$ (cf. Definition 2.4.3). Assume that $\mathcal{T} \neq \mathcal{T}_{0}(\mathcal{C})$ from now on. We start by remarking that in order to show that $\mathcal{T}=\mathcal{T}_{1}(\mathcal{C})$ (which is one of the possibilities), it would clearly suffice to build, for all $x \in \mathcal{C}$, an interval $I_{x}$ of $\mathcal{C}$ containing $x$ with $I_{x} \in \mathcal{T}$, and then to show that every subinterval of $I_{x}$ containing $x$ lies in $\mathcal{T}$ as well. Then, because $G$ is transitive on $\mathcal{T}$, it would suffice to do this for just one particular $x$. Letting $x$ be any point of $I=I_{x}$ in the statement just below, we thus see that the following equivalence holds,

FACT 2.5.2. - We have $\mathcal{T}=\mathcal{T}_{1}(\mathcal{C})$ if and only if there is an interval $I \in \mathcal{T}$ whose every subinterval also lies in $\mathcal{T}$.

Now, since $\mathcal{T}$ is distinct from $\mathcal{T}_{0}(\mathcal{C})$ it contains an interval whose boundary is nonempty; there are only two possibilities: 
First case. $-\mathcal{T}$ contains some bounded interval $I=] a, b[$ of $\mathcal{C}$. Pick any subinterval $] \alpha, \beta[$ of $I$. By transitivity of $G$ on $\mathcal{C}$, for every $r$ in $I$ there is an interval $J$ of $\mathcal{C}$ containing $a$ and a couple $(J, \phi)$ in $G$ with $\phi(a)=r$. Then the interval $I \cap J$ lies in $\mathcal{T}$, so that its image $J^{\prime}$ also does; the intersection $J^{\prime \prime}$ of that image with $I$, by construction, writes $\left.J^{\prime \prime}=\right] r, s[$ for some $s \in] r, b[$. Next considering the union of all the $J^{\prime \prime}$ which are obtained for some $r>\alpha$, we get that $] \alpha, b[\in \mathcal{T}$. By the same token, using $b$ instead of $a$, we construct ]$a, \beta[\in \mathcal{T}$; the intersection of $] a, \beta[$ and $] \alpha, b[$ being $] \alpha, \beta\left[\right.$, we have $\mathcal{T}=\mathcal{T}_{1}(\mathcal{C})$ in view of Fact 2.5.2.

Second case. - No bounded interval can be found in $\mathcal{T}$. Still, there is a couple of distinct intervals $I, J$ in $\mathcal{T}$ with $I \subset J$, otherwise we would have $\mathcal{T}=\mathcal{T}_{0}(\mathcal{C})$. With no loss of generality, we can assume that $J$ belongs to $\mathcal{T}_{0}(\mathcal{C})$ (that is: we can replace $J$ with the whole connected component of $I$ in $\mathcal{T}$ ). We now define the set $X_{-}$of all $r \in J$ for which $\mathcal{T}$ contains $]-\infty, r$ [ and the set $X_{+}$of all $r \in J$ for which $\mathcal{T}$ contains $] r,+\infty$ [ (we hope that the notation " $]-\infty, r[] r,,+\infty["$, which we haven't bothered to define, is self-evident enough). We have $X_{-} \cup X_{+} \neq \varnothing$, because by assumption the interval $I$ is bounded on one side and thus $X_{-} \cup X_{+}$must contain its bound.

Lemma 2.5.3. - If nonempty, $X_{-}$is an interval which is unbounded on the left; if nonempty, $X_{+}$is an interval which is unbounded on the right.

Proof. - Both cases are studied in the same way, so we do just one. Assume that $X_{+} \neq \varnothing$ and consider some $r \in X_{+}$. There is by transitivity of $G$, for any $s>r$, a couple $(U, \phi)$ in $G$ with $r \in U$ and $\phi(r)=s$. We must have $U=J$ or $U=] t,+\infty[$ for some $t<r$, otherwise we would have $U \cap] r,+\infty[$ both nonempty and bounded in $\mathcal{T}$, a contradiction. By the same token, since $\phi$ preserves the orientation and sends $r$ to $s$ we have $\phi(] r,+\infty[)=] s,+\infty[$, so that $s \in X_{+}$. In other words, $X_{+}$contains $s$ as soon as it contains some $r$ with $r<s$; this is a characterization of the open intervals which are unbounded on the right.

Lemma 2.5.4. - We have $X_{-} \cap X_{+}=\varnothing$ and $X_{-} \cup X_{+}=J$, so that by connexity of $J$, either $J=X_{-}$or $J=X_{+}$.

Proof. - First show that $X_{-} \cap X_{+}=\varnothing$ : indeed if some $r$ lies in both intervals then there is an $\alpha<r$ in $X_{+}$and a $\beta>r$ in $X_{-}$, which by definition of $X_{-}$and $X_{+}$satisfy $] \alpha,+\infty[\in \mathcal{T}$ and $]-\infty, \beta[\in \mathcal{T}$, so that their bounded intersection $] \alpha, \beta\left[\right.$ lies in $\mathcal{T}$; a contradiction. Next show that $X_{-} \cup X_{+}=J$ : indeed assume that some $r \in J$ would lie neither in $X_{-}$nor in $X_{+}$; then the only connected neighborhood of $r$ in $\mathcal{T}$ would be $J$. On the other hand, unless $X_{+}$is empty, we can find by transitivity of $G$ a couple $(U, \phi)$ in $G$ with $r \in U$ 
and $\phi(r) \in X_{+}$; by what we just saw, $U=J$. Let $s=\phi(r)$ and let $a<b<s$ lie in the interval $X_{+} \cap \phi(J)$ : then $\phi^{-1}(] b,+\infty[)$ is a subinterval of $J$ strictly contained in $\phi^{-1}(] a,+\infty[)$, so that $r$ possesses, after all, strictly more than one connected neighborhood in $\mathcal{T}$; a contradiction. Of course we reached that contradiction by assuming that $X_{+} \neq \varnothing$, but if $X_{+}=\varnothing$ we can perform the same proof mutatis mutandis with $X_{-}$instead of $X_{+}$(recall that $\left.X_{-} \cup X_{+} \neq \varnothing\right)$.

We know that $J=X_{-}$or $J=X_{+}$. This, given the definitions, means that $\mathcal{T}$ coincides with $\mathcal{T}(\vartheta)$ or with $\mathcal{T}(-\vartheta)$ in restriction to $J$. Now, let $J^{\prime}$ in $\mathcal{T}_{0}(\mathcal{C})$ be any connected component of $\mathcal{C}$; by transitivity of $G$, we can find an interval $I \in \mathcal{T}$ contained in $J$ and a couple $(I, \phi)$ in $G$ with domain $I$ and image $\phi(I) \subset J^{\prime}$. Then even if we have $\phi(I)=J^{\prime}$, we can always find a smaller interval $I^{\prime} \subset I, I^{\prime} \neq I$ in $\mathcal{T}$ and we will then have $\phi\left(I^{\prime}\right) \subset J^{\prime}$ with this time a strict inclusion. We can now repeat our construction on $J^{\prime}$, to conclude that $\mathcal{T}$ coincides with either $\mathcal{T}(\vartheta)$ or $\mathcal{T}(-\vartheta)$ in restriction to $J^{\prime}$ as well. Then, it may a priori happen that $\mathcal{T}$ coincides with $\mathcal{T}(\vartheta)$ on $J$ and with $\mathcal{T}(-\vartheta)$ on $J^{\prime}$ (say); but we can easily rule out this possibility by using the transitivity of $G$, which obviously implies that this pseudogroup can only leave invariant the two opposite orientations $\vartheta$ and $-\vartheta$.

\section{The geometry of a web}

3.1. Parallelizable webs. - For any $\kappa \in \mathbb{R}$, let $\mathcal{F}^{\kappa}$ denote the foliation of $\mathbb{R}^{2}$ by parallel lines with slope $\kappa$; let $\mathcal{F}^{\infty}$ be the foliation by vertical lines. For us, the model 3 -web $\mathcal{W}_{3}$ is by definition $\left(\mathcal{F}^{0}, \mathcal{F}^{\infty}, \mathcal{F}^{1}\right)$. Given any three pairwise distinct elements $\alpha, \beta$ and $\gamma$ of $\mathbb{R P}^{1}$, there will exist a homography $h$ sending the triple $(\alpha, \beta, \gamma)$ to $(0, \infty, 1)$; from there one quickly deduces that every parallelizable 3 -web is analytically conjugate to $\mathcal{W}_{3}$. Next, if $d \geq 4$ and $\delta_{4}, \ldots, \delta_{d}$ are pairwise distinct real numbers all different from 0 and 1 , we define the model $d$-web $\mathcal{W}_{d}\left(\delta_{4}, \ldots, \delta_{d}\right)$ as being $\left(\mathcal{F}^{0}, \mathcal{F}^{\infty}, \mathcal{F}^{1}, \mathcal{F}^{\delta_{4}}, \ldots, \mathcal{F}^{\delta_{d}}\right)$. According to Theorem 1.4.1, whose proof, as the reader may check, does not rely on the contents of this subsection, any parallelizable web is analytically conjugate to some model. So, the problem of describing the set-theoretic or analytic conjugacy classes of parallelizable webs amounts to that of describing the corresponding conjugacy classes for models. Then, the group of translations of $\mathbb{R}^{2}$ acts transitively on $\mathbb{R}^{2}$ and leaves invariant every model; so that two models will be conjugate precisely if they are conjugate by a map which fixes the origin. We are therefore left with the following

Problem 3.1.1. - Let $\mathcal{W}$ and $\mathcal{W}^{\prime}$ be two model d-webs. Under what conditions are $\mathcal{W}$ and $\mathcal{W}^{\prime}$ set-theoretically, or analytically, conjugate by a map $f$ fixing the origin? 
The analytic case is very simple to deal with:

Proposition 3.1.2. - Let $\mathcal{W}$ and $\mathcal{W}^{\prime}$ be as above. Then $\mathcal{W}$ and $\mathcal{W}^{\prime}$ are analytically conjugate if, and only if, they coincide.

Proof. - Both webs have the same first three foliations $\mathcal{F}^{0}, \mathcal{F}^{\infty}$ and $\mathcal{F}^{1}$. Then the exponents $\delta_{\imath}$ of the subsequent foliations $\mathcal{F}^{\delta_{\imath}}$, if any, can be defined as the (constant) cross-ratios of the tangents to $\mathcal{F}^{0}, \mathcal{F}^{\infty}, \mathcal{F}^{1}$ and $\mathcal{F}^{\delta_{2}}$ in that order; this shows that they are invariants of $C^{1}$-conjugacy.

Another way of stating the preceding proposition would be to say that there is exactly one analytic conjugacy class of parallelizable 3-webs, and that for $d \geq 4$ the "vector of slopes" $\left(\delta_{4}, \ldots, \delta_{d}\right)$ is a complete invariant of analytic conjugacy. In the set-theoretic context, the answer is somewhat different:

Proposition 3.1.3. - Let $\mathcal{W}$ and $\mathcal{W}^{\prime}$ be as in Problem 3.1.1 and have respective vectors of slopes $\left(\delta_{4}, \ldots, \delta_{d}\right)$ and $\left(\delta_{4}^{\prime}, \ldots, \delta_{d}^{\prime}\right)$. Then $\mathcal{W}$ and $\mathcal{W}^{\prime}$ are set-theoretically conjugate if, and only if, there is a field automorphism $\phi$ from the field $\mathbb{Q}\left(\delta_{4}, \ldots, \delta_{d}\right)$ to the field $\mathbb{Q}\left(\delta_{4}^{\prime}, \ldots, \delta_{d}^{\prime}\right)$ such that $\phi\left(\delta_{\imath}\right)=\delta_{\imath}^{\prime}$ for all $\imath \in\{4, \ldots, d\}$.

Proof. - First suppose that both fields are isomorphic, and build from there a set-theoretic conjugacy between the corresponding webs. For that, we choose some isomorphism $\phi$ from $\mathbb{Q}\left(\delta_{4}, \ldots, \delta_{d}\right)$ to $\mathbb{Q}\left(\delta_{4}^{\prime}, \ldots, \delta_{d}^{\prime}\right)$ satisfying $\phi\left(\delta_{\imath}\right)=\delta_{\imath}^{\prime}$ for all $\imath$, then choose a basis $\left\{b_{\imath}\right\}$ of the $\mathbb{Q}\left(\delta_{4}, \ldots, \delta_{d}\right)$-vector space $\mathbb{R}$, a basis $\left\{b_{\imath}^{\prime}\right\}$ of the $\mathbb{Q}\left(\delta_{4}^{\prime}, \ldots, \delta_{d}^{\prime}\right)$-vector space $\mathbb{R}$, and finally define a set-theoretic bijection $\alpha$ of $\mathbb{R}$ onto itself by the formula $\alpha\left(\sum k_{\imath} b_{\imath}\right)=\sum \phi\left(k_{\imath}\right) b_{\imath}^{\prime}$ where the $k_{\imath}$ belong to $\mathbb{Q}\left(\delta_{4}, \ldots, \delta_{d}\right)$ and both sums are understood to be finite (this makes sense because the families $\left(b_{\imath}\right)$ and $\left(b_{\imath}^{\prime}\right)$ are equipotent, so that we can use the same collection of indexes $\imath$ for both of them). Once this is done, it is straightforwardly checked that the map $f(x, y)=(\alpha(x), \alpha(y))$ conjugates $\mathcal{W}$ to $\mathcal{W}^{\prime}$. Notice that even in the case of a self-conjugacy from $\mathcal{W}$ to itself, a well-chosen $\alpha$ will not be continuous; therefore, it will not be measurable by Dufour's Theorem.

Conversely, suppose that $\mathcal{W}$ and $\mathcal{W}^{\prime}$ are set-theoretically conjugate by some map $f(x, y)=(\alpha(x, y), \beta(x, y))$. Then, first of all, $f$ leaves $\mathcal{F}^{0}$ invariant, so that $\beta$ does not depend on $x$; similarly, because $f$ leaves $\mathcal{F}^{\infty}$ invariant, $\alpha$ does not depend on $y$. Next, since $f$ leaves both the origin and $\mathcal{F}^{1}$ invariant, it preserves the diagonal $x=y$; at the same time, it sends it to the curve $\alpha(x)=\beta(y)$, so that the two maps $\alpha$ and $\beta$ from $\mathbb{R}$ to itself must coincide. Finally, consider some other leaf of $\mathcal{F}^{1}$, which admits the equation $y=x+k$ for a certain constant $k$ : its image by $f$ will be both the curve $y=\alpha^{-1}(\alpha(x)+k)$ and some other leaf of $\mathcal{F}^{1}$ with equation $y=x+k^{\prime}$; from which it is not hard to deduce 
that $\alpha(x+k)=\alpha(x)+\alpha(k)$. This takes place for every $x$ and $k$, so that $\alpha$ is an automorphism of the additive group $\mathbb{R}$; we now show the following

Lemma 3.1.4. - Let $\Gamma$ be the subset of couples $(a, b)$ in $\mathbb{R} \times \mathbb{R}$ for which $\alpha(a x)=b \alpha(x)$ for all $x \in \mathbb{R}$. Let $A$ and $B$ respectively be the first and the second projections of $\Gamma$ on $\mathbb{R}$. Then $A$ and $B$ are subfields of $\mathbb{R}$ and $\Gamma$ is the graph of an isomorphism of fields between $A$ and $B$.

Proof. - The relation $(a, b) \in \Gamma$ is, in any case, the graph of a bijection from $A$ to $B$, because this relation implies $\alpha(a)=b \alpha(1)$ where $\alpha$ is bijective and $\alpha(1) \neq 0=\alpha(0)$. Write $b=\phi(a)$ if $(a, b)$ lies in $\Gamma$. Since $\alpha(0)=0$ and $\alpha(x)=\alpha(x)$ we have $(0,0) \in \Gamma$ and $(1,1) \in \Gamma$. Because

$$
0=\alpha(x-x)=\alpha(x)+\alpha(-x)
$$

by additivity of $\alpha$, we have $(-1,-1) \in \Gamma$ too. Next, let $(a, b)$ and $\left(a^{\prime}, b^{\prime}\right)$ belong to $\Gamma$ : for every $x$, again by additivity of $\alpha$, we have

$$
\alpha\left(\left(a+a^{\prime}\right) x\right)=\alpha(a x)+\alpha\left(a^{\prime} x\right)=\left(b+b^{\prime}\right) \alpha(x),
$$

so that $\left(a+a^{\prime}, b+b^{\prime}\right)$ also lies in $\Gamma$; applying the definition, we also have $\alpha\left(a a^{\prime} x\right)=b \alpha\left(a^{\prime} x\right)=b b^{\prime} \alpha(x)$, so that $\left(a a^{\prime}, b b^{\prime}\right)$ lies in $\Gamma$ too. Finally, if $a$ is nonzero then we deduce from $\alpha(x)=\alpha\left(a a^{-1} x\right)=b \alpha\left(a^{-1} x\right)$ that $\left(a^{-1}, b^{-1}\right)$ lies in $\Gamma$. To sum up, both $A$ and $B$ are stable by inversion, addition, multiplication, opposition (which is multiplication by -1 ) and $\phi$ moreover commutes with addition and multiplication, as asserted.

Resume showing Proposition 3.1.3. Let $\imath$ be some index in $\{4, \ldots, d\}$ and consider the line $y=\delta_{\imath} x$ which is the leaf of $\mathcal{F}^{\delta_{\imath}}$ passing through the origin: its image under $f$ is the curve $y=\alpha^{-1}\left(\delta_{\imath} \alpha(x)\right)$, and at the same time it is the leaf of $\mathcal{F}^{\delta_{\imath}^{\prime}}$ passing through the origin - that is to say, the line $y=\delta_{\imath}^{\prime} x$. From there we deduce that $\alpha\left(\delta_{\imath} x\right)=\delta_{\imath}^{\prime} \alpha(x)$ for all $x$. This shows that the subfields $A$ and $B$ in Lemma 3.1 .4 respectively contain $\mathbb{Q}\left(\delta_{4}, \ldots, \delta_{d}\right)$ and $\mathbb{Q}\left(\delta_{4}^{\prime}, \ldots, \delta_{d}^{\prime}\right)$; according to said lemma, there is a field isomorphism $\phi$ from $A$ to $B$ with the property that $\phi\left(\delta_{\imath}\right)=\delta_{\imath}^{\prime}$ for all $\imath \in\{4, \ldots, d\}$.

To sum up all that precedes:

- Between every pair of parallelizable set-theoretically conjugate webs, there exists a nonmeasurable conjugacy;

- Still, all parallelizable 3 -webs are analytically conjugate (that is, if we take Theorem 1.4.1 for granted);

- Finally if $d \geq 4$, there exist set-theoretically conjugate $d$-webs which are not even measurably conjugate, whence Theorem 1.4.2. 
An example of the last situation is provided by the family of models $\mathcal{W}\left(\delta_{4}, \ldots, \delta_{d}\right)$ for which the extension $\mathbb{Q}\left(\delta_{4}, \ldots, \delta_{d}\right)$ of $\mathbb{Q}$ is purely transcendental with transcendence degree $d-3$ (any two models of that kind are set-theoretically conjugate!)

3.2. Conjugacies and continuity. - Let $\mathcal{W}=\left(\mathcal{F}_{1}, \ldots, \mathcal{F}_{d}\right)$ be a $d$-web with $d \geq$ 3. We can associate with $\mathcal{W}$ the $d$ curves $\mathcal{C}_{\imath}=\mathcal{C}\left(\mathcal{F}_{\imath}\right)$; for a better understanding of what follows, one should keep in mind that $\mathcal{C}_{\imath}$ is just $\mathbb{R}^{2}$ equipped with some strange topology. Now, if $\mathcal{W}^{\prime}$ is another $d$-web, we can define as well the curves $\mathcal{C}_{\imath}^{\prime}$ for it; then if $f$ is a set-theoretic conjugacy from $\mathcal{W}$ to $\mathcal{W}^{\prime}$, it will be psychologically useful to distinguish between $f$ when seen as a map from $\mathbb{R}^{2}$ to $\mathbb{R}^{2}$ and the same $f$ when seen as a map from $\mathcal{C}_{\imath}$ to $\mathcal{C}_{\imath}^{\prime}$ (although both objects are logically the same): this is why we will use the notation $f_{\imath}$ in the second case. This convention, while logically useless, makes the proofs all the more legible: e.g., if we say " $f_{\imath}$ is continuous", we mean that $f_{\imath}\left(\mathcal{T}_{1}\left(\mathcal{C}_{\imath}\right)\right)=\mathcal{T}_{1}\left(\mathcal{C}_{\imath}^{\prime}\right)$. We next remark that the maps $f_{1}, \ldots, f_{d}$ are continuous as soon as $f$ is so; what is not so trivial is that the reciprocal also holds true:

Proposition 3.2.1. - The function $f$ is analytic if and only if one of the $f_{i}$ 's is continuous.

Proof. - With no loss of generality, we may assume that $\imath=1$ during the proof. We first show the following, seemingly weaker

LEMmA 3.2.2. - The function $f$ is continuous if and only if both $f_{1}$ and $f_{2}$ are so.

Proof. - Let $I_{1}$ and $I_{2}$ be nonempty subsets of $\mathbb{R}^{2}$; we assume that each $I_{\imath}$ lies in a single leaf $L_{i}$ of the corresponding foliation $\mathcal{F}_{i}$. Notice that $L_{1}$ cannot meet $L_{2}$ at more than one point: indeed, let $\phi$ be a map from $\mathbb{R}^{2}$ to $\mathbb{R}$ whose level curves are the $\mathcal{F}_{1}$-leaves (it exists by Kaplan's Theorem). By transversality, $\phi$ will be strictly monotonous on $L_{2}$, while by definition it will be constant on $L_{1}$. So, it will be constant and injective at the same time in restriction to $L_{1} \cap L_{2}$.

Now, given $P_{1} \in I_{1}$ and $P_{2} \in I_{2}$, the $\mathcal{F}_{2}$-leaf of $P_{1}$ and the $\mathcal{F}_{1}$-leaf of $P_{2}$ intersect at most at one point; if this point exists, we call it $S\left(P_{1}, P_{2}\right)$. Conversely, for any point $Q$ of $\mathbb{R}^{2}$, the $\mathcal{F}_{3-\imath}$-leaf of $Q$ meets $I_{\imath}$ at one point $P_{\imath}(Q)$ at most; and if both $P_{1}(Q)$ and $P_{2}(Q)$ are defined, we obviously have $Q=S\left(P_{1}(Q), P_{2}(Q)\right)$. All this to say that we can define an injective function $S\left(P_{1}, P_{2}\right)$ from part of $I_{1} \times I_{2}$ to $\mathbb{R}^{2}$ in a purely set-theoretic way; when, and only when, $S$ is defined on all of $I_{1} \times I_{2}$, we call its image the square with sides $I_{1}$ and $I_{2}$ for $\mathcal{W}$. 
The following picture helps understand the construction: we identify $\mathbb{R}^{2}$ with the inside of a euclidian triangle thanks to some analytic map; on that triangle, we let $\mathcal{W}$ be the 3 -web by segments which are parallel to one of the triangle's sides. Assume $\mathcal{F}_{1}$ and $\mathcal{F}_{2}$ are respectively the horizontal and the vertical foliation: then $I_{1}$ and $I_{2}$ allow us to build a square, while $I_{1}^{\prime}$ and $I_{2}^{\prime}$ do not (to the leftmost point of $I_{1}^{\prime}$ and the highest point of $I_{2}^{\prime}$, there corresponds no point of the triangle).

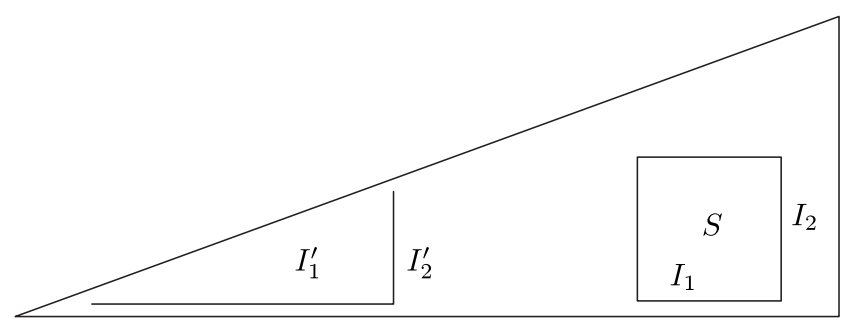

Picture 1: squares for webs

Letting $\Sigma(\mathcal{W})$ denote the collection of all squares for $\mathcal{W}$, we have obviously $f(\Sigma(\mathcal{W}))=\Sigma\left(\mathcal{W}^{\prime}\right)$; furthermore, with an obvious choice of notation, we will have

$$
f \circ S\left(P_{1}, P_{2}\right)=S\left(f_{1}\left(P_{1}\right), f_{2}\left(P_{2}\right)\right)
$$

for any square $S$ for $\mathcal{W}$. Consider now the special case where $I_{1}$ and $I_{2}$ are intervals: this provides us with a special subfamily $\Sigma_{0}(\mathcal{W})$ of squares which are domains in $\mathbb{R}^{2}$; notice that the map $S\left(P_{1}, P_{2}\right)$ from $I_{1} \times I_{2}$ to $\mathbb{R}^{2}$ is in that case a bianalytic map.

LEMMA 3.2.3. - Every $P \in \mathbb{R}^{2}$ has a neighborhood $\mathcal{N}$ which is a square for $\mathcal{W}$.

Proof. - Define $\mathcal{F}_{1}$ and $\mathcal{F}_{2}$ as the foliations by level curves of analytic submersions $\phi_{1}$ and $\phi_{2}$ from $\mathbb{R}^{2}$ to $\mathbb{R}$. Then, the map $\phi(Q)=\left(\phi_{1}(Q), \phi_{2}(Q)\right)$ is a local diffeomorphism from $\mathbb{R}^{2}$ to itself which sends the 2 -web $\left(\mathcal{F}_{1}, \mathcal{F}_{2}\right)$ to that whose leaves are the vertical and horizontal lines. Letting $(\alpha, \beta)$ stand for $\phi(P)$, there will exist for $\varepsilon>0$ small enough a neighborhood $\mathcal{N}$ of $P$ in restriction to which $f$ is a diffeomorphism onto the square $\sup (|x-\alpha|,|y-\beta|)<\varepsilon$. By construction, that $\mathcal{N}$ has the desired property.

Assume now that $f_{1}$ and $f_{2}$ both are continuous: then from (1) and the fact that $S$ is bianalytic, it follows at once that $f$ is continuous on the image $S\left(I_{1}, I_{2}\right)$ of $S$. So, Lemma 3.2.2 is just a consequence of the existence of sufficiently many squares ensured by Lemma 3.2.3. 
Getting back to Proposition 3.2.1, we will show that $f_{2}$ is continuous as soon as $f_{1}$ has the same property. It suffices to do so in the neighborhood of every point $P \in \mathbb{R}^{2}$. Respectively call $L_{1}$ and $L_{2}$ the $\mathcal{F}_{1}$-leaf and the $\mathcal{F}_{2}$-leaf of $P$; let $P^{\prime}, L_{1}^{\prime}, L_{2}^{\prime}$ be respectively $f\left(P^{\prime}\right), f\left(L_{1}^{\prime}\right), f\left(L_{2}^{\prime}\right)$. We define a bianalytic map $\phi$ from $L_{1}$ to $L_{2}$, defined in some neighborhood of $P$, and a bianalytic map $\phi^{\prime}$ from $L_{1}^{\prime}$ to $L_{2}^{\prime}$, defined in some neighborhood of $P^{\prime}$, for which $f_{2} \circ \phi=\phi^{\prime} \circ f_{1}$. This clearly suffices to ensure the desired result. Since both definitions are symmetric, we will define only the first of these two maps by letting $Q_{2}=\phi\left(Q_{1}\right)$ if $Q_{1} \in L_{1}, Q_{2} \in L_{2}$ and $Q_{1}$ and $Q_{2}$ are on the same $\mathcal{F}_{3}$-leaf. The following picture illustrates this.

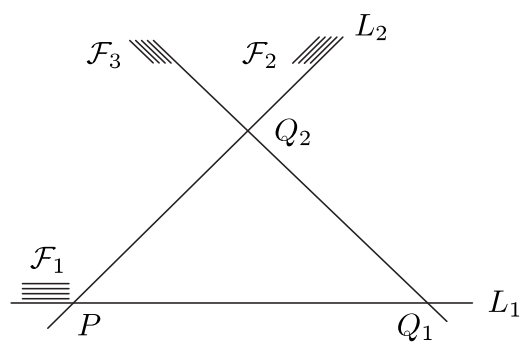

Picture 2: proof of 3.2.1

We have shown that $f$ is continuous as soon as $f_{1}$ is so; and by Dufour's Theorem, $f$ is analytic as soon as it is continuous.

3.3. The pseudogroups of a web. - Let $k$ be some index in $\{1, \ldots, d\}$. We will now define a certain pseudogroup $G_{k}$ on $\mathcal{C}_{k}$ which emerges from $\mathcal{W}$ through certain purely set-theoretic considerations; as a result, the map $f_{k}$ will yield a conjugacy between $G_{k}$ and the corresponding pseudogroup $G_{k}^{\prime}$ on $\mathcal{C}_{k}^{\prime}$. This fact will allow us to show Theorem 1.4.1.

Let $L$ and $L^{\prime}$ be any two leaves of $\mathcal{F}_{k}$, pick some index $\imath$ in $\{1, \ldots, d\}-\{k\}$ and define the subset $X^{\imath}\left(L, L^{\prime}\right)$ of $L \times L^{\prime}$ consisting of those couples $(P, Q)$ which lie along the same $\mathcal{F}_{\imath}$-leaf.

In the following picture, we identify $\mathbb{R}^{2}$ with the interior of a euclidian triangle and let $\mathcal{W}$ be some linear web with $\mathcal{F}_{1}$ horizontal and $\mathcal{F}_{2}$ vertical. Let $k=1$ and $\imath=2$ : then with our choice of leaves $L, L_{1}^{\prime}$ and $L_{2}^{\prime}$, we have $X^{2}\left(L, L_{2}^{\prime}\right)=\varnothing$ while $X^{2}\left(L, L_{1}^{\prime}\right)$ is an unbounded interval.

LEMMA 3.3.1. - If nonempty, $X^{\imath}\left(L, L^{\prime}\right)$ is the graph of a bianalytic map $\tau^{\imath}\left(L, L^{\prime}\right)$ whose domain is a subinterval of $L$ and whose image is a subinterval of $L^{\prime}$. 


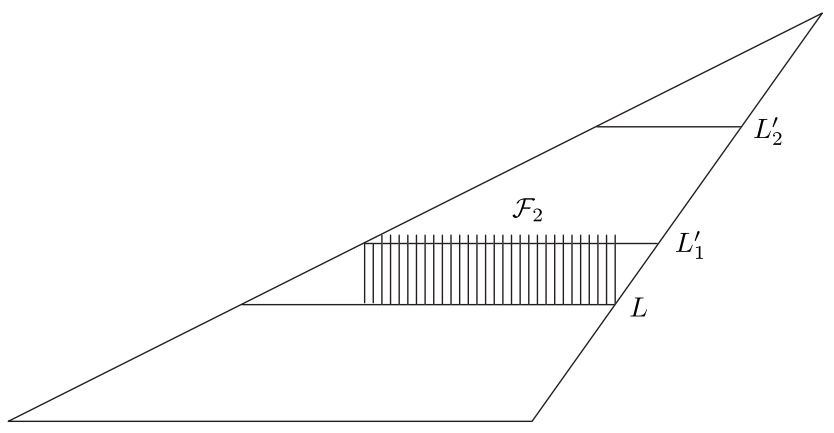

Picture 3: the domain of $X^{i}\left(L, L^{\prime}\right)$ may be empty

Proof. - An $\mathcal{F}_{\imath}$-leaf can only meet an $\mathcal{F}_{k}$-leaf at one point (as we saw during the proof of Lemma 3.2.2); therefore, if nonempty, $X^{\imath}\left(L, L^{\prime}\right)$ is the graph of an injective map $\tau^{\imath}\left(L, L^{\prime}\right)$ from part of $L$ to part of $L^{\prime}$. Openness of the domain and bianalyticity come at once from the implicit function theorem and transversality of $\mathcal{F}_{k}$ with $\mathcal{F}_{i}$; the fact that $\tau^{\imath}\left(L, L^{\prime}\right)$ has intervals for its domain and target comes from elementary topological considerations based on the fact that any leaf of any foliation cuts $\mathbb{R}^{2}$ in two topological disks.

We call $\tau^{\imath}\left(L, L^{\prime}\right)$ a translation of $\mathcal{F}_{k}$ along $\mathcal{F}_{i}$; we notice that $\tau^{\imath}\left(L, L^{\prime}\right)$ has $\tau^{\imath}\left(L^{\prime}, L\right)$ for inverse. We call bounce of $L$ on $L^{\prime}$ along $\mathcal{F}_{\imath}$ and $\mathcal{F}_{\jmath}$ the map from $L$ to $L$ which writes as $B_{\imath}^{\jmath}\left(L ; L^{\prime}\right)=\tau^{\jmath}\left(L^{\prime}, L\right) \circ \tau^{\imath}\left(L, L^{\prime}\right)$; the inverse of $B_{\imath}^{\jmath}\left(L ; L^{\prime}\right)$ is $B_{\jmath}^{\imath}\left(L ; L^{\prime}\right)$. The set $\tau_{k}$ of all possible translations $\tau^{\imath}\left(L, L^{\prime}\right)$, where $L, L^{\prime}$ are leaves of $\mathcal{F}_{k}$ and $\imath$ is an index in $\{1, \ldots, d\}-\{k\}$, generates a pseudogroup $G_{k}$ on $\mathcal{C}_{k}$. We can similarly define a pseudogroup $G_{k}^{\prime}$ on $\mathcal{C}_{k}^{\prime}$. Now, even if $G_{k}$ and $G_{k}^{\prime}$ consist of analytic maps, their definition from $\mathcal{W}$ and $\mathcal{W}^{\prime}$ only uses set-theoretic notions, so that we will have, as planned:

Proposition 3.3.2. - $f_{k}$ yields a set-theoretic conjugacy from $G_{k}$ to $G_{k}^{\prime}$.

Picture 4 may surely help, and justify at the same time our heuristic use of the noun "bounce".

Let $L, L^{\prime}$ and $L^{\prime \prime}$ be leaves of $\mathcal{F}_{k}$. Let $\imath, \jmath$ be indexes other than $k$. We notice for further use the following equalities, which are obvious on our picture:

$$
\begin{gathered}
\tau^{\imath}\left(L^{\prime}, L^{\prime \prime}\right) \circ \tau^{\imath}\left(L, L^{\prime}\right)=\tau^{\imath}\left(L, L^{\prime \prime}\right), \\
\tau^{\jmath}\left(L^{\prime}, L^{\prime \prime}\right) \circ \tau^{\imath}\left(L, L^{\prime}\right)=\tau^{\jmath}\left(L, L^{\prime \prime}\right) \circ B_{\imath}^{\jmath}\left(L, L^{\prime}\right) .
\end{gathered}
$$

We next check that $G_{k}$ satisfies the assumption of Lemma 2.5.1 (and of course, a similar result holds for $G_{k}^{\prime}$ as well). 


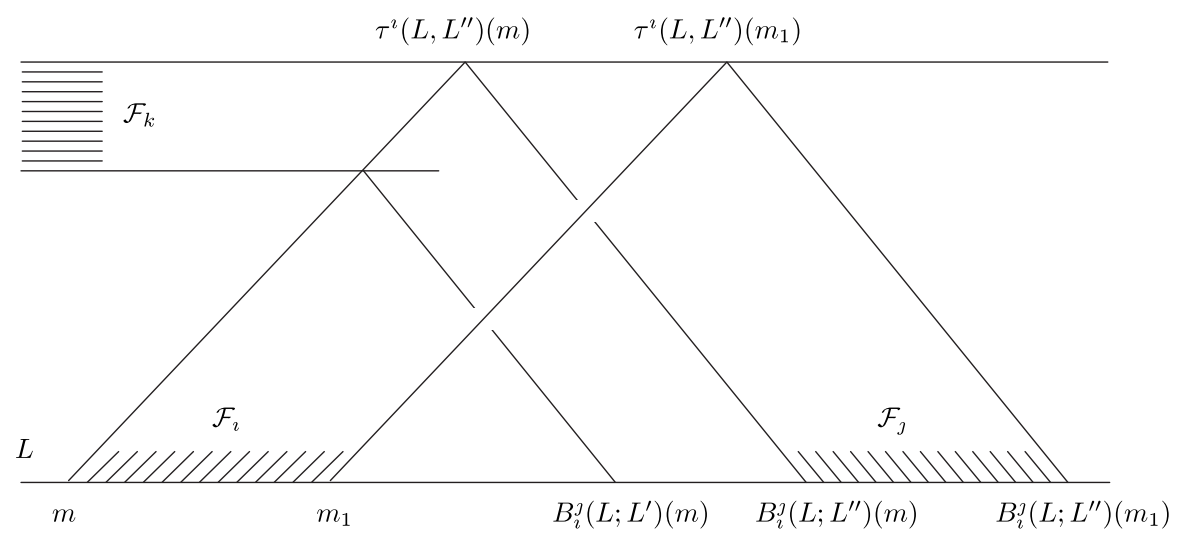

Picture 4: translations and bounces in a web

Proposition 3.3.3. - The pseudogroup $G_{k}$ is transitive on $\mathcal{C}_{k}$ and preserves the natural orientation of that curve given by the choice of some nonvanishing vector field tangent to $\mathcal{F}_{k}$.

Proof. - $G_{k}$ preserves the orientation because it is spanned by maps which do so (we hope this is obvious enough on Picture 4). Check that it is also transitive. For that, pick some point $P$ in $\mathcal{C}_{k}$ (which, we recall one more time, is $\mathbb{R}^{2}$ as a set). Also choose two distinct indexes $\imath, \jmath$ in $\{1, \ldots, d\}-\{k\}$ and notice that the orbit of $P$ under $G_{k}$ is invariant by both the foliations $\mathcal{F}_{\imath}$ and $\mathcal{F}_{\jmath}$, which are transverse: therefore, this orbit is open in the usual topology of the plane. Since every orbit is open and the plane is connected, there is just one orbit.

3.4. A disjonction of cases. - We now notice that webs fall into three classes corresponding to different behaviours of the pseudogroups $G_{k}$ we just built.

A. Incomplete webs. - We call $\mathcal{W}$ complete if for any pair of distinct indexes $\imath, \jmath \in\{1, \ldots, d\}$, every leaf $L_{\imath}$ of $\mathcal{F}_{\imath}$ and every leaf $L_{\jmath}$ of $\mathcal{F}_{\jmath}$ meet at exactly one point (we already saw that they met at most at one point in any case). Otherwise, we call $\mathcal{W}$ incomplete. If $\mathcal{W}$ is incomplete, then by applying a suitable permutation on the set $\{1, \ldots, d\}$ of indexes we may assume with no loss of generality that some leaf $L_{1}$ of $\mathcal{F}_{1}$ and some leaf $L_{2}$ of $\mathcal{F}_{2}$ do not meet. We then notice that in such a case, the subset $\mathcal{X}$ of $\mathbb{R}^{2}$ consisting of points whose $\mathcal{F}_{2}$-leaf does not meet $L_{1}$ is a nonempty closed subset whose complementary open subset is also nonempty: therefore, we may assume with no loss of generality that $L_{2}$ lies in the boundary of $\mathcal{X}$. Consider next some point $P$ in $L_{2}$ and its leaf $L_{1}^{\prime}$ for $\mathcal{F}_{1}$ : since $L_{1}^{\prime}$ is transverse to the boundary of $\mathcal{X}$, part of $L_{1}^{\prime}$ will lie outside $\mathcal{X}$, to the effect that the translation $\tau^{2}\left(L_{1}, L_{1}^{\prime}\right)$ 
will not have empty definition domain. On the other hand, this map will not be a bijection from $L_{1}$ to $L_{1}^{\prime}$, because by construction its image will not contain $P$ (see Picture 5). We thus have shown the following

Proposition 3.4.1. - If $\mathcal{W}$ is incomplete, then we may assume with no loss of generality that $G_{1}$ contains a certain couple $(U, \phi)$ where $U$ is not a leaf of $\mathcal{F}_{1}$.

In the following picture, we identify $\mathbb{R}^{2}$ with the Poincaré half-plane; $\mathcal{F}_{1}$ is the foliation by half-lines which originate from some boundary point $A$, similarly the leaves of $\mathcal{F}_{2}$ come from another point $B$, and $\mathcal{F}_{3}$ consists of horizontal lines.

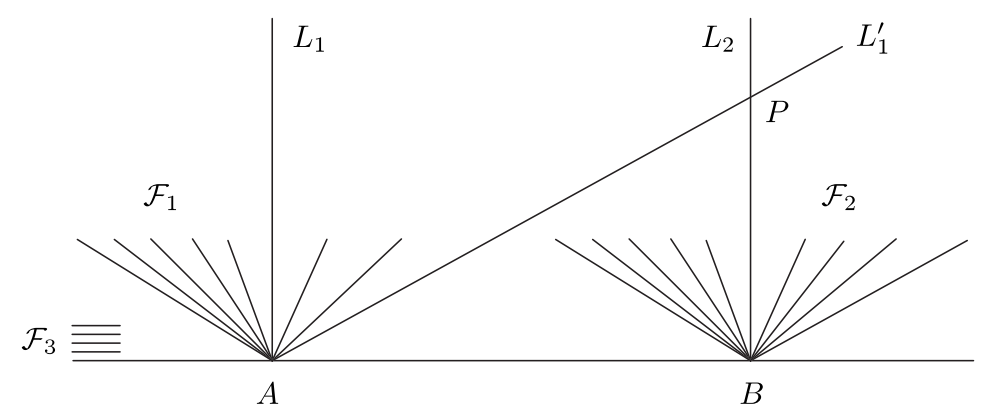

Picture 5: $\tau^{2}\left(L_{1}, L_{1}^{\prime}\right)$ sends all $L_{1}$ to $] A P[$

From our last proposition, we deduce that incomplete webs are rigid:

Proposition 3.4.2. - $\mathcal{W}$ is complete if and only if $\mathcal{W}^{\prime}$ is so, and if these webs are not complete then $f_{1}$ is continuous.

Proof. - The fact that both $\mathcal{W}$ and $\mathcal{W}^{\prime}$ must be complete or incomplete at the same time is obvious in view of the definition. Next, let $\mathcal{T}$ be the weakest topology on $\mathcal{C}_{1}$ which is both $G_{1}$-invariant and not weaker than $\mathcal{T}_{0}\left(\mathcal{C}_{1}\right)$ : if $\mathcal{W}$ is incomplete then $\mathcal{T}$ is spanned by intervals one at least of which does not belong to $\mathcal{T}_{0}\left(\mathcal{C}_{1}\right)$ (that interval is the " $U$ " of Proposition 3.4.1). In that case we may apply Lemma 2.5.1 to see that $\mathcal{T}$ belongs to the space $\mathcal{G}\left(\mathcal{C}_{1}\right)$ of good topologies on $\mathcal{C}_{1}$. Defining a similar topology $\mathcal{T}^{\prime}$ on $\mathcal{C}_{1}^{\prime}$, we will have $f_{1}(\mathcal{T})=\mathcal{T}^{\prime}$ and therefore $f_{1}\left(\mathcal{G}\left(\mathcal{C}_{1}\right)\right) \cap \mathcal{G}\left(\mathcal{C}_{1}^{\prime}\right) \neq \varnothing$, whence the continuity of $f_{1}$ by Lemma 2.2.4. 
B. Complete, curved webs. - Let $\mathcal{W}$ be complete. Then, the stabilizer $G_{k}^{L}$ in $G_{k}$ of any leaf $L$ of $\mathcal{F}_{k}$ is a pseudogroup whose every map has $L$ for both its domain and target; in other words, it is a group of homeomorphisms of $L$ in the usual sense.

Lemma 3.4.3. - The group $G_{k}^{L}$ is a connected subgroup of $\operatorname{Hom}(L)$ for the compact-open topology.

Proof. - It suffices to show that $G_{k}^{L}$ admits as a set of generators the collection of all bounces $B_{\imath}^{\jmath}\left(L ; L^{\prime}\right)$, with $L^{\prime}$ a variable leaf of $\mathcal{F}_{k}$ and $\imath, \jmath$ indexes different from $k$ and from one another. Indeed, assume for a moment that this result holds. For the compact-open topology on $\operatorname{Hom}(L)$, and for fixed $\imath$ and $\jmath$, the bounce $B_{\imath}^{\jmath}\left(L ; L^{\prime}\right)$ obviously depends continuously on the leaf $L^{\prime}$, which in turn evolves in the connected space of leaves of $\mathcal{F}_{k}$; therefore, if we let $\mathfrak{B}_{\imath}^{\jmath}(L)$ denote the space of all bounces $B_{\imath}^{\jmath}\left(L ; L^{\prime}\right)$, that space is connected. Next notice that all the $\mathfrak{B}_{\imath}^{\jmath}(L)$ contain the identity map of $L$ (which writes $B_{\imath}^{\jmath}(L ; L)$ ). The union of connected spaces with a common point is connected; therefore the union $\mathfrak{B}(L)$ of the $\mathfrak{B}_{\imath}^{J}(L)$ is connected. A topological group with a connected system of generators has to be connected; so if $\mathfrak{B}(L)$ generates $G_{k}^{L}$, that group will be connected.

Show that $\mathfrak{B}(L)$ generates $G_{k}^{L}$. Any element $w$ in $G_{k}^{L}$ writes as a "word" in the "alphabet" of translations: more precisely, there exist an integer $n \geq 1$, a family of leaves $L_{1}, \ldots, L_{n}$ in $\mathcal{F}_{k}$ and a sequence of indexes $a_{1}, \ldots, a_{n+1} \in$ $\{1, \ldots, d\}-\{k\}$ such that

$$
w=\tau^{a_{n+1}}\left(L_{n}, L\right) \circ \tau^{a_{n}}\left(L_{n-1}, L_{n}\right) \circ \cdots \circ \tau^{a_{2}}\left(L_{1}, L_{2}\right) \circ \tau^{a_{1}}\left(L, L_{1}\right) .
$$

We will assume that $n$ is the smallest integer for which such a decomposition of $w$ exists. We already noticed that for all leaves $A, B, C$ of $\mathcal{F}_{k}$, one has

$$
\tau^{\imath}(B, C) \circ \tau^{\imath}(A, B)=\tau^{\imath}(A, C)
$$

so, the assumption that $n$ is minimal implies that $a_{p} \neq a_{p+1}$ for $1 \leq p \leq n$. We now reason by induction on $n$. Notice that $n$ is not the minimum number of translations required to write $w$ : that number is $n+1$. If $n=1$ then $w=B_{a_{1}}^{a_{2}}\left(L ; L_{1}\right)$ and we are done. If $n>1$, notice that

$$
w=w^{\prime} \circ \tau^{a_{2}}\left(L_{1}, L_{2}\right) \circ \tau^{a_{1}}\left(L, L_{1}\right)
$$


where $w^{\prime}$ is the product of $n-1$ translations. We saw that

$$
\tau^{a_{2}}\left(L_{1}, L_{2}\right) \circ \tau^{a_{1}}\left(L, L_{1}\right)=\tau^{a_{2}}\left(L, L_{2}\right) \circ B_{a_{1}}^{a_{2}}\left(L ; L_{2}\right)
$$

so that $w$ is the product of the bounce $B_{a_{1}}^{a_{2}}\left(L ; L_{2}\right)$ and of the word $w^{\prime} \circ \tau^{a_{2}}\left(L, L_{2}\right)$. But since that word lies in $G_{k}^{L}$ and is the product of $n$ translations at most, it writes as a product of bounces by the assumption of our induction: therefore, $w$ does too.

We will say that $\mathcal{W}$ is curved if $G_{k}^{L}$ is not simply transitive on $L$ for some $L$ and some $k$. If $\mathcal{W}$ is not curved we call it flat. Recall that a classical invariant, called the Blaschke (or Chern) curvature, has been defined for 3-webs; and although this is not obvious at the time being, the contents of the next subsection imply that the 3-webs which are curved in our sense are precisely those whose curvature tensor is not everywhere vanishing - which is our reason for choosing this terminology.

Let $L$ be as above, let $P$ belong to $L$ and let $G_{k}^{P}$ be the stabilizer of $P$ in $G_{k}$, which is a subgroup of $G_{k}^{L}$. We have the following

LEMma 3.4.4. $-G_{k}^{P}$ is a connected group.

Proof. - Since $G_{k}$ is transitive on $\mathcal{C}_{k}, G_{k}^{L}$ has to be transitive on $L$; so that the map from $G_{k}^{L}$ to $L$ sending $g$ to $g(P)$ endows $G_{k}^{L}$ with the structure of a principal bundle with group $G_{k}^{P}$ (acting on $G_{k}^{L}$ by translations on the right) and contractible base space $L$. Bundles with contractible base spaces are product bundles, so that $G_{k}^{L}$ has the same homotopy type as $G_{k}^{P}$; since $G_{k}^{L}$ is connected, we have the desired result.

From there we deduce:

Proposition 3.4.5. - If $\mathcal{W}$ is curved then $f_{k}$ is continuous for some $k$.

Proof. - We assume that $\mathcal{W}$ is curved, which implies that $G_{k}^{L}$ is not simply transitive for some $k$ and $L$ as above; or in other words, that $G_{k}^{P}$ is not trivial for some $k$ and $P$ as above. Consider now the family of $G_{k}^{P}$-orbits in $L$ : by assumption, not every point of $L$ is fixed under $G_{k}^{P}$, so that certain of these orbits will have more than one point; let $\mathcal{O}$ be one of them. Since $\mathcal{O}$ is an orbit of a connected group acting continuously on a line, and $\mathcal{O}$ does not consist of a global fixed point, $\mathcal{O}$ has to be an interval in $L$. This interval is not equal to $L$ because it does not contain $P$. We now let $\mathcal{T}$ be the weakest topology on $\mathcal{C}_{k}$ which contains every orbit such as $\mathcal{O}$, for every possible $P$. This is by construction a convenient, $G_{k}$-invariant topology on $\mathcal{C}_{k}$ which is distinct from $\mathcal{T}_{0}\left(\mathcal{C}_{k}\right)$; its image by $f_{k}$ is a topology of the same nature on $\mathcal{C}_{k}^{\prime}$, and we can conclude as in Proposition 3.4.2. 
C. Complete, flat webs. - We will show the following

Proposition 3.4.6. - If $\mathcal{W}$ is both complete and flat, then $\mathcal{W}^{\prime}$ is so, and in that case, both webs are analytically parallelizable.

Proof. - The first part of the statement is obvious. We next choose two isomorphisms of Lie groups $x \rightarrow g_{1}(x)$ and $x \rightarrow g_{2}(x)$ from $\mathbb{R}$ to $G_{1}^{P}$ and $G_{2}^{P}$ respectively, where $P$ is some point of $\mathbb{R}^{2}$ chosen as an origin. We write respectively $L_{1}$ and $L_{2}$ for the $\mathcal{F}_{1}$ - and the $\mathcal{F}_{2}$-leaf of $P$. Let $Q$ be any point of $\mathbb{R}^{2}$ : since $\mathcal{W}$ is complete, the $\mathcal{F}_{2}$-leaf of $Q$ meets $L_{1}$ at one point exactly, and this point writes $g_{1}(x) P$ in a unique way; similarly, the $\mathcal{F}_{1}$-leaf of $Q$ meets $L_{2}$ at one point exactly, and this point writes $g_{2}(y) P$ in a unique way. The map $Q \mapsto(x, y)$ provides us with an analytic global chart in which we work from now on; this leads us to assume with no loss of generality that $\mathcal{F}_{1}=\mathcal{F}^{0}$, that $\mathcal{F}_{2}=\mathcal{F}^{\infty}$, that $P$ is the origin, that $L_{1}$ and $L_{2}$ are the coordinate axes and that $G_{1}^{P}$ and $G_{2}^{P}$ are the groups of translations along these axes. Consider now some index $\imath \in\{3, \ldots, d\}$, some parameter $c$ and two leaves $A$ and $B$ of $\mathcal{F}_{i}$ : these leaves will intersect the leaf $L_{1}(c)$ of $\mathcal{F}_{1}$ defined by $y=c$ at points whose coordinates in our chart write respectively in the form $(a(c), c)$ and $(b(c), c)$. Computing explicitly the values that the bounce $B_{\imath}^{2}\left(L_{1}, L_{1}(c)\right)$ from $L$ to itself takes for $x=a(0)$ and $x=b(0)$, and remembering that this bounce is by assumption a translation in $x$, we conclude that $a(c)-b(c)=a(0)-b(0)$. This happens for all values of $c$ and all leaves $A, B$ of $\mathcal{F}_{\imath}$; therefore, $\mathcal{F}_{\imath}$ is invariant by horizontal translations. The same argument with $\mathcal{F}_{1}$ and $\mathcal{F}_{2}$ exchanged allows us to conclude that $\mathcal{W}$ is invariant by all the translations of $\mathbb{R}^{2}$, which of course forces this web to be a model.

3.5. From continuity to analyticity. - Once $f$ has been shown to be continuous, it will be analytic by Dufour's Theorem. To conclude this paper, we would like to hint to an alternative proof of that fact, on the lines of an argument by Nakai. We just sketch the construction, because this part of the result is not really original. Let $P$ be a point, and show that $f$ is analytic on some neighborhood of $P$. If this neighborhood is small enough, the restriction of $\mathcal{F}_{1}$ to it is the family of level curves for some analytic submersion $\phi$; next, define locally each of $\mathcal{F}_{2}$ and $\mathcal{F}_{3}$ as the foliations by orbits of a (possibly not complete) vector field $X_{\imath}$, normalized by the condition $\mathrm{d} \phi\left(X_{\imath}\right)=1$. Finally, let $X_{1}=X_{3}-X_{2}$. Since $\mathrm{d} \phi\left(X_{1}\right)=\mathrm{d} \phi\left(X_{3}\right)-\mathrm{d} \phi\left(X_{2}\right)=0$, we see that $X_{1}$ is everywhere tangent to $\mathcal{F}_{1}$; since $\mathcal{F}_{2}$ and $\mathcal{F}_{3}$ are transverse, $X_{1}$ is nowhere vanishing. Local integration of the ODE $\dot{x}=X_{1}(x)$ starting from some point $P$ yields a local chart for $\mathcal{C}_{1}$ around $P$. If two such charts overlap, the transition map from one to another is a translation; but we can consider if we wish the bigger atlas $\mathfrak{A}$ on $\mathcal{C}_{1}$ which contains all the charts above and is at the same

TOME $135-2007-\mathrm{N}^{\circ} 1$ 
time invariant by affine transformations of its parameters - in other words, we turn $\mathcal{C}_{1}$ into an affine curve thanks to $X_{1}$. This amounts to dropping the arbitrariness in our choice of $X_{1}$ by considering all the charts corresponding to all possible fields $X_{1}$; for given the normalization $\mathrm{d} \phi\left(X_{\imath}\right)=1$, any other field $X_{1}^{\prime}$ such as $X_{1}$ writes in the form $X_{1}^{\prime}=g \cdot X_{1}$ with $g$ a first integral for $\mathcal{F}_{1}$. We have:

Proposition 3.5.1. - The affine atlas $\mathfrak{A}$ described above for $\mathcal{C}_{1}$ is an invariant of $C^{0}$-conjugacy.

In other words: the map $f_{1}$ from $\mathcal{C}_{1}$ to $\mathcal{C}_{1}^{\prime}$ equipped with the atlases above will be an affine map as soon as it is continuous!

No proof, but a hint. - Pick a leaf $L$ of $\mathcal{F}_{1}$. Let $I$ be an interval in $L$; let $B_{n}$ be a sequence of bounces from $L$ to $L$ which are all defined on $I$, and assume that $B_{n}$ tends to the identity on $I$ for the compact-open topology. Let $\Gamma_{n}$ be the pseudogroup on $L$ which is spanned by $B_{n}$; finally, let $\Gamma\left(I, B_{n}\right)$ be the "limit" of the sequence $\Gamma_{n}$ of pseudogroups, in the following sense:

Definition 3.5.2. - A continuous injective map $g$ from $I$ to $L$ lies in $\Gamma\left(I, B_{n}\right)$ if some sequence $\gamma_{n} \in \Gamma_{n}$ admits $g$ for an accumulation point w.r.t. the compact-open topology on the space of continuous maps from $I$ to $L$.

Perform this construction for all possible $L, I$ and $B_{n}$. Consider the pseudogroup $G$ spanned by the union of all the $\Gamma\left(I, B_{n}\right)$ thus obtained. Using only elementary calculus and the equalities

$$
\mathrm{d} f_{1}\left(X_{2}\right)=1, \quad \mathrm{~d} f_{1}\left(X_{3}\right)=1, \quad X_{1}=X_{3}-X_{2},
$$

one can show that $G$ is transitive on $L$ and that its elements are restrictions to suitable intervals of the elements of the pseudoflow of $X_{1}$. Thus, we defined this analytic pseudoflow - of which the affine structure of $\mathcal{C}_{1}$ derives - by purely topological considerations.

\section{BIBLIOGRAPHY}

[1] W. BlaschKe - Einführung in die Geometrie der Waben, BirkhaüserVerlag, 1955.

[2] W. Blaschke \& G. Bol - Geometrie der Gewebe, Springer, 1938.

[3] É. CARTAN - "Les sous-groupes des groupes continus de transformations", Ann. École Norm. Sup. 25 (1908), p. 57-124.

[4] S. S. Chern - "Web geometry", Bull. Amer. Math. Soc. 6 (1982), p. 1-8.

[5] J.-P. Dufour - "Rigidity of webs", in Web Theory and Related Topics, World Scientific, 2001. 
[6] J.-P. Dufour \& P. Jean - "Rigidity of webs and families of hypersurfaces, Singularities and dynamical systems (Iráklion, 1983)", NorthHolland Math. Stud., vol. 103, 1985, p. 271-283.

[7] A. Haefliger \& G. Reeb - "Variétés (non séparées) à une dimension et structures feuilletées du plan", Enseign. Math. 3 (1957), p. 107-125.

[8] M. Herman - "Sur la conjugaison différentiable des difféomorphismes du cercle à des rotations", Publ. Math. Inst. Hautes Études Sci. 49 (1979), p. $5-233$.

[9] W. KaPLAN - "Regular curve-families filling the plane, I", Duke Math. J. 7 (1940), p. 154-185.

[10] _ , "Regular curve-families filling the plane, II", Duke Math. J. 8 (1941), p. 11-46.

[11] R. MAÑÉ - Ergodic theory and differentiable dynamics, Ergeb. Math. Grenzgeb., vol. 8, Springer, 1987.

[12] I. NAKAI - "Topology of complex webs of codimension one and geometry of projective space curves", Topology 26 (1987), p. 475-504.

[13] _ "A naive guide to web geometry", in Web Theory and Related Topics, World Scientific, 2001. 